\title{
Temperature and Stretching Effects on Complementarity Determining Regions (CDRs) Conformation and Stability of Nimotuzumab $\mathrm{F}(\mathrm{ab})$-Fragment
}

\author{
T.S. Humani ${ }^{1,2^{*}}$, M.A. Martoprawiro ${ }^{2}$ and A. Mutalib ${ }^{3}$ \\ ${ }^{I}$ Center for Radioisotopes and Radiopharmaceutical Technology, National Nuclear Energy Agency, \\ Puspiptek Area, Serpong Tangerang 15310, Indonesia \\ ${ }^{2}$ Department of Computational Science, Faculty of Science, Bandung Institute of Technology, \\ Jl. Ganesha 10, Bandung 40132, Indonesia \\ ${ }^{3}$ Department of Chemistry, Faculty of Mathematics and Natural Science, Padjajaran University, \\ Jl Raya Bandung Sumedang Km 21 Jatinangor, Sumedang 45361, Indonesia
}

\section{ARTICLE INFO}

Article history:

Received 04 October 2014

Received in revised form 13 March 2015

Accepted 23 March 2015

Keywords:

Nimotuzumab

$\mathrm{F}(\mathrm{ab})$-Nimotuzumab

Complementarity Determining Regions (CDRs)

Unfolding

Molecular Dynamics

Steered Molecular Dynamics

\begin{abstract}
A B S T R A C T
Nimotuzumab is a humanized monoclonal antibody $(\mathrm{mAb})$, a potential anticancer against epidermal growth factor receptor (EGFR)overexpressed by glioma, head and neck, lung, ovarium, and colon cancers. The combination of its use with both external and internal beam radiotherapies showed improvement of the therapeutic effect. However, the high molecular weight slows its uptake on tumor cells. In a recent development, nimotuzumab has been fragmented and then labeled using diagnostic and therapeutic radionuclides, such as gallium-68, yttrium-90, lutetium177, and holmium-166. In that preparation, nimotuzumab is often conditioned in various environments with variations of $\mathrm{pH}$, temperature and the presence of other compounds. In this research, molecular dynamics (MD) simulation have been carried out to study the CDRs conformational change of nimotuzumab due to the effect of temperature, and also steered molecular dynamics (SMD) simulation to study the stability of nimotuzumab domain as a result of external forces. The simulations were performed using the Not Just Another Molecular Dynamics (NAMD) program package and the analysis was performed with the Visual Molecular Dynamics (VMD) program package. Based on the stability analysis of each residue on the heavy chain, the active site (CDR3 region) that is at residues numbered 98 (Tryptophan) and 99 (Phenylalanine) has the highest conformational changes. On the light chain, the change occurs at residues numbered 1 (Aspartat), 127 (Serin), and 186 (Tyrosine); and that none of that residues is part of active site or CDRs region of the light chain. The SMD simulation was carried out by fixing the $\mathrm{N}$-terminal end of the heavy chain and applying external forces to the $\mathrm{C}$-terminal end. The pulling was set at a constant velocity of $0.5 \AA / p s$. The force peak arising at the beginning of the unfolding process is $1226 \mathrm{pN}$. This force was allegedly caused by the rupture of hydrogen bonds between the heavy chain residue VAL211 (Valine) and the heavy chain residue TYR194 (Tyrosine).
\end{abstract}

\section{INTRODUCTION}

Cancer represents one of the leading causes of death in the world and lung cancer is the most common cause of cancer-related death worldwide,

\footnotetext{
* Corresponding author.

E-mail address: humani@batan.go.id

DOI: http://dx.doi.org/10.17146/aij.2015.351
}

in which non-small cell lung cancer reaches $75 \%$ of all lung cancer cases [1]. According to WHO, in 2008 there were 7.5 million deaths caused by cancer, $70 \%$ of which occurred in developing countries. Because of the high number of deaths caused by cancer, various studies of clinical treatments of cancer therapy have been conducted. 
However, the widely and commonly used therapies by means of surgery, radiotherapy, and chemotherapy have still not provided very satisfactory results, particularly in addition to some adverse side effects to the cancer patients [2]. Much ongoing research is directed to targeted therapy using biomolecules, such as monoclonal antibodies, which are able to specifically interact with target molecules, such as receptors and antigens, which are overexpressed by cancer cells. The mechanism of action of monoclonal antibodies is usually preceded by their interaction through non-covalent bonds with the receptors on the surface of cancer cell membranes in the body. It appears that the cell surface of non-small cell lung cancer overexpress Epidermal Growth Factor Receptor (EGFR), as well as breast cancer, glioma, colon cancer, and head and neck cancer [3,4]. The EGFR belongs to the family of tyrosine kinase receptors (RTKs). Overexpression of EGFR is often associated with cancer malignancy and plays a significant role in tumor development and progression, including cell proliferation, regulation of apoptotic cell death, angiogenesis, and metastatic spread [5]. Therefore, one of the methods used for the cancer therapy is to inhibit the effects of EGFR activation, with the aim of blocking tumor growth and invasion, using either specific antibodies directed against its ligand-binding domains or small molecules inhibiting its tyrosine kinase activity [6-9].

Nimotuzumab or also called $\mathrm{h}-\mathrm{R} 3$ is a humanized monoclonal antibody specific to EGFR and is one of the alternative cancer therapeutic agents. Although nimotuzumab is not included in the 12 antibodies which have been approved by the Food and Drug Administration (FDA), its use would be interesting because of the absence of side effects such as skin rashes [3,10]. This is in contrast to the use of other of monoclonal antibodies targeting EGFR which cause skin rashes in every use $[11,12]$.

Over the past several years, metalradionuclide-labeled nimotuzumab has been developed as target-specific radiopharmaceuticals that is commonly called radioimmunotherapeutic agents or radioimmunodiagnostic agents if labeled with therapeutic or diagnostic radionuclides, respectively, using a bifunctional chelating agent (BFCA). The BFCA could form both stable metal radionuclide complexes and immunoconjugates with antibodies and peptides. In general, the process of conjugation and labeling of monoclonal antibodies can be carried out in various conditions such as $\mathrm{pH}$ adjustment and temperature changes [13]. This condition would certainly impact the three-dimensional structure of nimotuzumab as a protein. Accordingly, it is necessary to computationally observe the effects prior to both labeling with radionuclides and conjugation with nimotuzumab. Ideally, both labeling and conjugation processes will not much affect the conformation of nimotuzumab, particularly the residues in the CDR that play a main role in the binding to the receptors.

The purpose of this study was to computationally investigate (i) the effect of temperature changes on the three dimensional conformation of the nimotuzumab $F(a b)$ fragment, especially the residues on the Complementarity Determining Regions (CDRs), (ii) the residues that play role in maintaining the stability of the structure of the nimotuzumab $\mathrm{F}(\mathrm{ab})$ fragment, and (iii) the minimal force to trigger the unfolding process of the nimotuzumab $\mathrm{F}(\mathrm{ab})$ fragment including a bond that establishes the stability of the unfolding process, using molecular dynamics simulations. This computational study is very important to predict the behavior of the CDRs of nimotuzumab or their fragments prior to binding affinity studies of them to EGFR using either molecular docking or radioligand binding assay.

\section{EXPERIMENTAL METHODS}

The program packages used to perform molecular dynamics simulations and steered molecular dynamics simulations (SMD) were Not Just Another Molecular Dynamics (NAMD) program [14], and the Visual Molecular Dynamics (VMD) program for the preparation of the input files [15], respectively. The NAMD and VMD programs are distributed for free and available at http://www.ks.uiuc.edu/Research.

A Pdb file $(3 \mathrm{GKW})$ of the nimotuzumab $\mathrm{F}(\mathrm{ab})$ fragment that contains the atomic coordinates and velocities of atoms in the system was obtained from the protein data bank in http://www.pdb.org. The fragment used in this study has two chains, namely the heavy chain and the light chain. The active regions or the so-called Complementarity Determining Regions (CDRs) are divided into six active regions, namely CDR1, CDR2, and CDR3 which are located in the heavy chain, and CDR1, CDR2, and CDR3 in the light chain (see Fig. 1). 


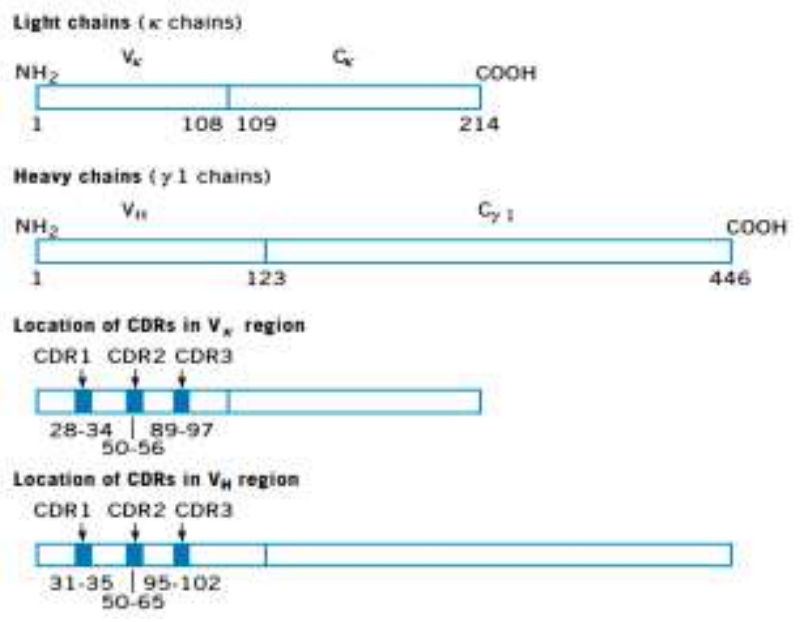

Fig. 1. Complementarity Determining Regions (CDRs) of a monoclonal antibody IgG. Taken from www.piercenet.com on September 15th, 2013.

NAMD was run using a CHARMM force field and TIP3 solvent. The first step of simulation studies was the preparation of molecules, which includes reading pdb files and psf files, creation of boundary conditions, solvation and ionization. The next step was the energy minimization to reach a state with a minimum local energy, heating/ cooling gradually until the desired temperature, and equilibration until the energy converges to a certain value, then followed by the molecular dynamics and steered molecular dynamics (SMD) simulations. In this study, molecular dynamics simulations were run using the timestep of 2 fs, the cut-off of $20 \AA$, and a switching function at $10 \AA$ at the temperatures of $277 \mathrm{~K}, 310 \mathrm{~K}, 350 \mathrm{~K}$ and $373 \mathrm{~K}$, respectively. The temperatures of $277 \mathrm{~K}$ and $310 \mathrm{~K}$ represent conditions at which the nimotuzumab $\mathrm{F}(\mathrm{ab})$ fragment undergoes a conjugation reaction with a BFCA and labeling with a radionuclide, respectively. Steered molecular dynamics simulation (SMD) was performed by introducing external force at the C-terminal end of the nimotuzumab $\mathrm{F}(\mathrm{ab})$ fragment's heavy chain, whereas the N-terminal end was fixed. Simulations were performed using two protocols, i.e. with constant velocity and with constant force. In the first one, pulling was done at a constant velocity of $0.5 \AA / p s$ and $1 \AA / p s$. Force obtained in the first protocol then will be used as a reference force for the second protocol.

The simulation results were analyzed using VMD program that focused on the threedimensional conformational changes of the nimotuzumab $F(a b)$ fragment. The analysis was based on the change in the value of the root mean square displacement (RMSD) or root mean square deviation of the atoms after the simulation process compared with the initial position (reference). RMSD is defined as:

$$
R M S D=\sqrt{\frac{1}{N} \sum_{i=1}^{N}[r(i, t)-r(i, 0)]^{2}}
$$

where $N$ is the number of atoms in the protein and $r(i, t)$ is the position of the $i$-th atom at time $t$.

\section{RESULTS AND DISCUSSION}

\section{Molecular dynamics simulation of nimotuzumab in various temperatures}

The difference of temperature effect on the nimotuzumab $\mathrm{F}(\mathrm{ab})$ fragment structure can be described by the Root Mean Square Displacement (RMSD) values of $\mathrm{C} \alpha$ atoms that refer changes in the conformation of each residue. Analysis of RMSD during the $900 \mathrm{ps}$ simulation at a temperature of $277 \mathrm{~K}$, $310 \mathrm{~K}, 350 \mathrm{~K}$, and $373 \mathrm{~K}$, obtained RMSD values of $2.37,2.50,2.46$ and 2.53 , respectively (Fig. 2).

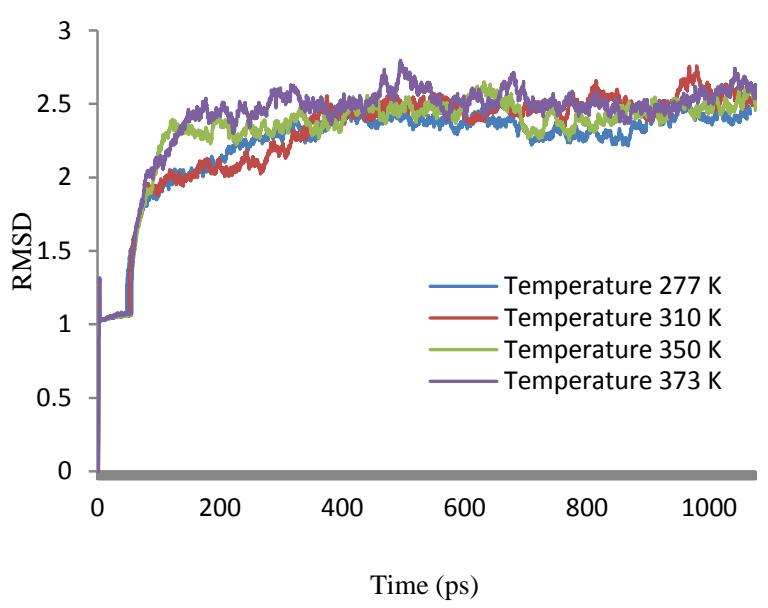

Fig. 2. Graph of RMSD versus time simulated at a various temperatures.

The RMSD values for all temperatures show that the change in temperature affects the structure of a protein. However, the RMSD analysis of $\mathrm{C} \alpha$ atoms only describes the global stability of the nimotuzumab $\mathrm{F}(\mathrm{ab})$ fragment structure. Therefore, in addition to global stability, the local one is necessary to be studied, especially to the stability of the active regions of a protein. Figure 3 depicted RMSD analysis for each residue of the heavy chain (see also Fig. 4). 


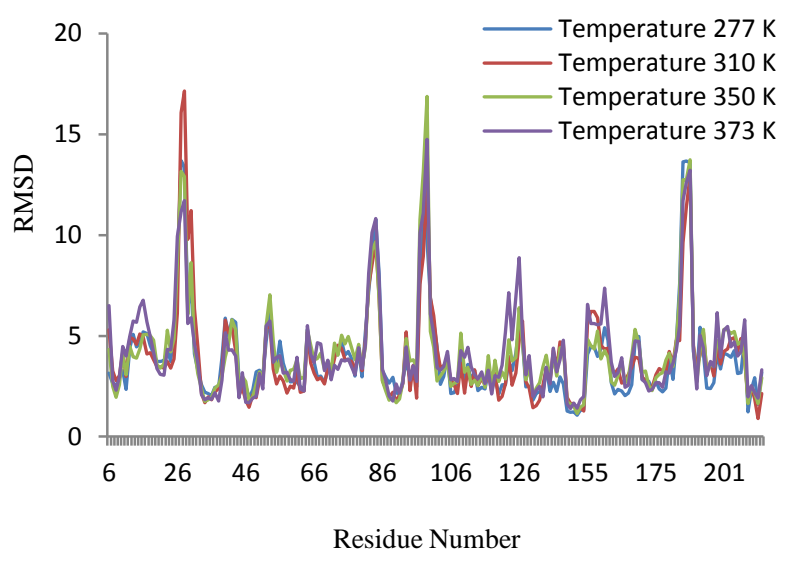

Fig. 3. Graph of RMSD per residue of the heavy chain at temperatures of $277 \mathrm{~K}, 310 \mathrm{~K}, 350 \mathrm{~K}$ and $373 \mathrm{~K}$.

Increasing the temperature has a considerable effect, especially toward residues numbered 27 (tyrosine), 28 (threonine), 83 (arginine), 84 (serine), 98 (tryptophan), 99 (phenylalanine), 183 (threonine), 184 (valine), and 185 (proline) as well.

Table 1 shows that residues numbered 98 (tryptophan) and 99 (phenylalanine) have the highest RMSD value (bold numbered in the table). Both of these residues are in the active region of the CDR3 region of the heavy chain of the nimotuzumab $\mathrm{F}(\mathrm{ab})$ fragment, while in the CDR1 and CDR2 regions, conformational changes are lower.

Table 1. RMSD of CDRs at heavy chains

\begin{tabular}{|c|c|c|c|c|c|}
\hline \multirow{2}{*}{$\begin{array}{l}\text { Residue } \\
\text { Number }\end{array}$} & \multicolumn{4}{|c|}{ Temperature } & \multirow{2}{*}{$\begin{array}{l}\text { Active } \\
\text { Regions }\end{array}$} \\
\hline & $277 \mathrm{~K}$ & $310 \mathrm{~K}$ & $350 \mathrm{~K}$ & $373 \mathrm{~K}$ & \\
\hline 31 & 4.09826 & 6.35544 & 4.39998 & 4.59832 & \multirow{5}{*}{ CDR1 } \\
\hline 32 & 3.31039 & 4.48606 & 3.12978 & 3.65685 & \\
\hline 33 & 2.57676 & 2.13008 & 2.3719 & 2.08144 & \\
\hline 34 & 2.19873 & 1.68624 & 1.74045 & 1.82967 & \\
\hline 35 & 2.13463 & 1.96555 & 1.81716 & 1.93581 & \\
\hline 50 & 3.29149 & 2.79883 & 3.21107 & 3.1205 & \multirow{16}{*}{ CDR2 } \\
\hline 51 & 2.96262 & 2.66188 & 2.80788 & 2.36553 & \\
\hline 52 & 4.72147 & 5.35354 & 5.5147 & 5.49044 & \\
\hline 53 & 6.20754 & 5.55378 & 7.04405 & 5.72595 & \\
\hline 54 & 4.03009 & 3.35364 & 4.76277 & 3.67969 & \\
\hline 55 & 3.59234 & 2.6026 & 3.19416 & 3.92815 & \\
\hline 56 & 4.75153 & 3.01297 & 3.79746 & 4.01523 & \\
\hline 57 & 3.65281 & 2.74907 & 3.33643 & 3.14433 & \\
\hline 58 & 3.04487 & 2.14835 & 2.7568 & 3.1875 & \\
\hline 59 & 2.71378 & 2.51741 & 3.30179 & 2.81572 & \\
\hline 60 & 2.7341 & 2.4096 & 3.34061 & 2.75258 & \\
\hline 61 & 2.69814 & 3.26195 & 3.81485 & 3.94231 & \\
\hline 62 & 2.48246 & 2.20732 & 2.88986 & 2.36917 & \\
\hline 63 & 2.32883 & 2.25302 & 2.92216 & 2.26935 & \\
\hline 64 & 5.32329 & 5.19484 & 5.2716 & 5.51224 & \\
\hline 65 & 4.22324 & 3.64015 & 4.53848 & 4.08544 & \\
\hline 95 & 3.34142 & 3.53301 & 3.81817 & 3.51976 & \multirow{8}{*}{ CDR3 } \\
\hline 96 & 2.47042 & 1.91743 & 3.43179 & 2.73598 & \\
\hline 97 & 8.11146 & 7.56012 & 10.7586 & 10.108 & \\
\hline 98 & 11.5956 & 8.92937 & 13.1247 & 11.0784 & \\
\hline 99 & 9.72212 & 12.7386 & 16.8761 & 14.745 & \\
\hline 100 & 6.87264 & 6.89802 & 5.22259 & 6.07626 & \\
\hline 101 & 5.67103 & 5.99849 & 4.42606 & 5.08585 & \\
\hline 102 & 4.46513 & 4.34767 & 2.75966 & 3.42783 & \\
\hline
\end{tabular}

Talavera et al. [10] reported that a residue tryptophan H103 plays an important role in nimotuzumab function as a monoclonal antibody. The RMSD values of tryptophan H103 (H refers to heavy chain) during the simulation period at temperatures of $277 \mathrm{~K}, 310 \mathrm{~K}, 350 \mathrm{~K}$ and $373 \mathrm{~K}$ in our study are $2.59,3.42,3.01$ and 3.30 , respectively. This implies that the conformation of $\mathrm{H} 103$ will be affected by temperature changes. Analysis of conformational changes was also carried out for the residues of $\mathrm{H} 103, \mathrm{H} 98$ and $\mathrm{H} 99$ at temperatures of $277 \mathrm{~K}$ and $310 \mathrm{~K}$ as depicted in Fig. 4. The conformation of the residue $\mathrm{H} 98$ at $277 \mathrm{~K}$ was more exposed to solvent than at $310 \mathrm{~K}$. On the contrary, conformation of the residue $\mathrm{H} 99$ was more opened at temperatures of $310 \mathrm{~K}$ compared to that of $277 \mathrm{~K}$. The conformation differences of residues of $\mathrm{H} 98$ and $\mathrm{H} 99$ at temperatures of $277 \mathrm{~K}$ and $310 \mathrm{~K}$ are very interesting to be considered due to the conjugation process of the nimotuzumab $F(a b)$ fragment which is commonly performed at a temperature of $277 \mathrm{~K}$. The probability of the conjugation reaction will be high at the residue $\mathrm{H} 98$ because of its smaller steric hindrance and larger solvent-accessible surface at $277 \mathrm{~K}$. This will be slightly different at the body temperature of $310 \mathrm{~K}$ due to slight changes of $\mathrm{H} 98$ conformation that leads to the different effectiveness of nimotuzumab as an anti-EGFR drug.
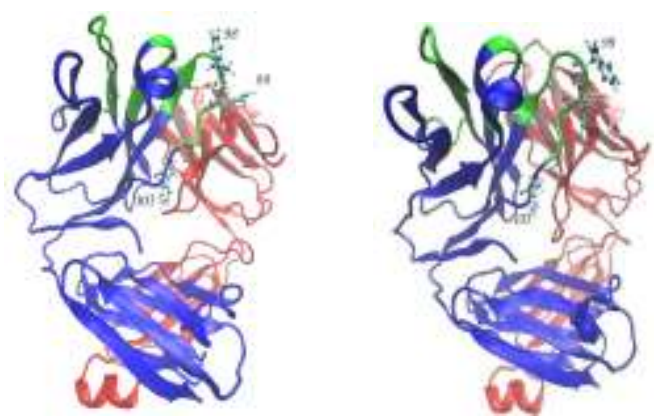

Fig. 4. Three dimensional conformation of residues of H98, $\mathrm{H} 99$, and $\mathrm{H} 103$ in nimotuzumab structure, left is at a temperature of $277 \mathrm{~K}$, right is at $310 \mathrm{~K}$.

On the other hand, residue H103 has a higher steric hindrance at temperature $310 \mathrm{~K}$ than that of $277 \mathrm{~K}$ due to the interaction between residue $\mathrm{H} 103$ and residues of 41-44 from the nimotuzumab $F(a b)$ fragment's light chain (see Fig. 4). Table 2 shows the effect of temperature on each residue. The numbers in bold in the Table 2 are the residues that have the highest RMSD value of each peak as shown also in Fig. 3. The grey line represents the residues with the highest RMSD that tend toward a different pattern. It appears that the highest conformational changes at temperatures of $310 \mathrm{~K}$, $350 \mathrm{~K}$ and $373 \mathrm{~K}$ were identified as residues of 
99 and 185. However, at a temperature of $277 \mathrm{~K}$, the highest conformational changes were residues of 98 and 184, one unit less than the changes experienced by the residues of 99 and 185 .

Table 2. Residues of high RMSD at several temperatures

\begin{tabular}{ccccc}
\hline \multirow{2}{*}{$\begin{array}{c}\text { Residue } \\
\text { Number }\end{array}$} & \multicolumn{4}{c}{ Temperature } \\
\cline { 2 - 5 } & $\mathbf{2 7 7}$ K & $\mathbf{3 1 0 ~ K}$ & $\mathbf{3 5 0} \mathbf{~ K}$ & $\mathbf{3 7 3 ~ K}$ \\
\hline 27 & $\mathbf{1 3 . 6 8 9}$ & 16.06 & $\mathbf{1 3 . 1 5 7}$ & 11.156 \\
28 & 13.392 & $\mathbf{1 7 . 1 5 5}$ & 12.902 & $\mathbf{1 1 . 6 9 8}$ \\
83 & 8.9024 & 8.5199 & 9.2354 & 10.116 \\
84 & $\mathbf{1 0 . 7 7 6}$ & $\mathbf{9 . 6 8 0 6}$ & $\mathbf{9 . 6 6 3 8}$ & $\mathbf{1 0 . 8 1 8}$ \\
$\mathbf{9 8}$ & $\mathbf{1 1 . 5 9 6}$ & 8.9294 & 13.125 & 11.078 \\
99 & 9.7221 & $\mathbf{1 2 . 7 3 9}$ & $\mathbf{1 6 . 8 7 6}$ & $\mathbf{1 4 . 7 4 5}$ \\
183 & 13.623 & 9.6149 & 12.746 & 11.711 \\
$\mathbf{1 8 4}$ & $\mathbf{1 3 . 6 6 4}$ & 11.379 & 12.736 & 12.601 \\
185 & 13.584 & $\mathbf{1 2 . 9 4 4}$ & $\mathbf{1 3 . 7 2 4}$ & $\mathbf{1 3 . 2 2}$ \\
\hline
\end{tabular}

The stability of three-dimensional conformations of the active site (CDRs) needs to be maintained in order for protein to still function normally. Figure 5 shows the RMSD per residue of the light chain of the nimotuzumab $\mathrm{F}(\mathrm{ab})$ fragment at temperatures of $277 \mathrm{~K}, 310 \mathrm{~K}, 350 \mathrm{~K}$, and $373 \mathrm{~K}$. It appears that the effect of temperature changes on the light chain of the nimotuzumab $\mathrm{F}(\mathrm{ab})$ fragment did not result in much change to the structure. However, significant changes occur in the residues numbered 1 (aspartate), 127 (serine), and 186 (tyrosine) as shown in Fig. 5. It can be inferred that all residues in the CDR1 (28-34), CDR2 (50-56), and CDR3 (89-97) have very small RMSD values (see Fig. 5).

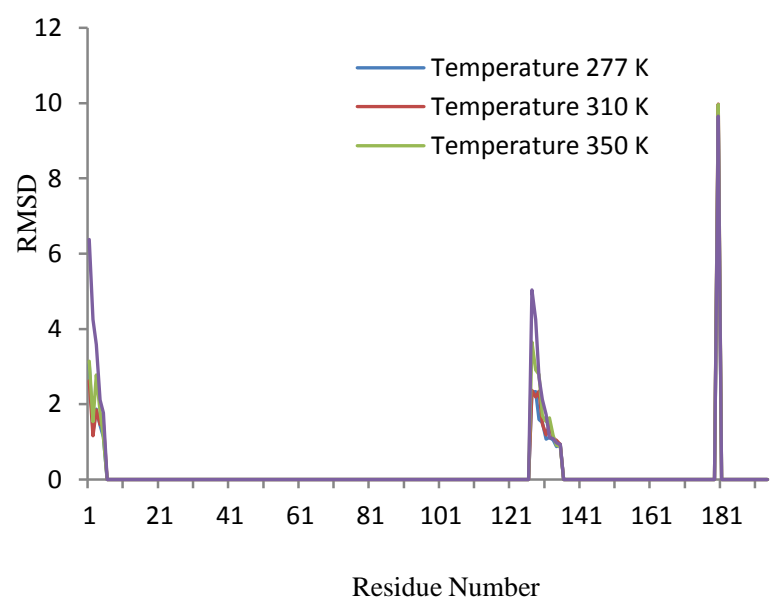

Fig. 5. RMSD per residue of nimotuzumab light chain at temperatures of $277 \mathrm{~K}, 310 \mathrm{~K}, 350 \mathrm{~K}$, and $373 \mathrm{~K}$.

In general, RMSD analysis shows that temperature changes more strongly affect the conformation of the nimotuzumab F(ab) fragment's heavy chain than that of the light chain.

\section{Steered Molecular Dynamics (SMD) simulations}

The purpose of SMD simulations is to determine both the structural stability of the nimotuzumab $F(\mathrm{ab})$ fragment that experiences external force and the bonds that affect that stability. SMD simulations were directed at observing the occurrence of the unfolding process of each domain in the nimotuzumab $\mathrm{F}(\mathrm{ab})$ fragment $(\mathrm{VL}, \mathrm{VH}, \mathrm{CL}$ and $\mathrm{CH} 1$ ). The first protocol of SMD simulation was performed by pulling the nimotuzumab $\mathrm{F}(\mathrm{ab})$ fragment with constant velocity of $0.5 \AA / \mathrm{ps}$ and $1 \AA / p s$ at the end of the C-terminal of the heavy chain, whereas the $\mathrm{N}$-terminal end of the heavy chain was fixed.

The SMD simulation applied for the heavy chain results in the force peak of $1226 \mathrm{pN}$ for the pulling velocity of $0.5 \AA / \mathrm{ps}$ and $1688 \mathrm{pN}$ for the pulling velocity $1 \AA /$ ps, at 14 ps (Fig. 6). Analysis of hydrogen bonds in this period indicates that ruptures and formations of hydrogen bonds occur. The total number of hydrogen bonds that occur during the 900 ps simulated was about 699 bonds.

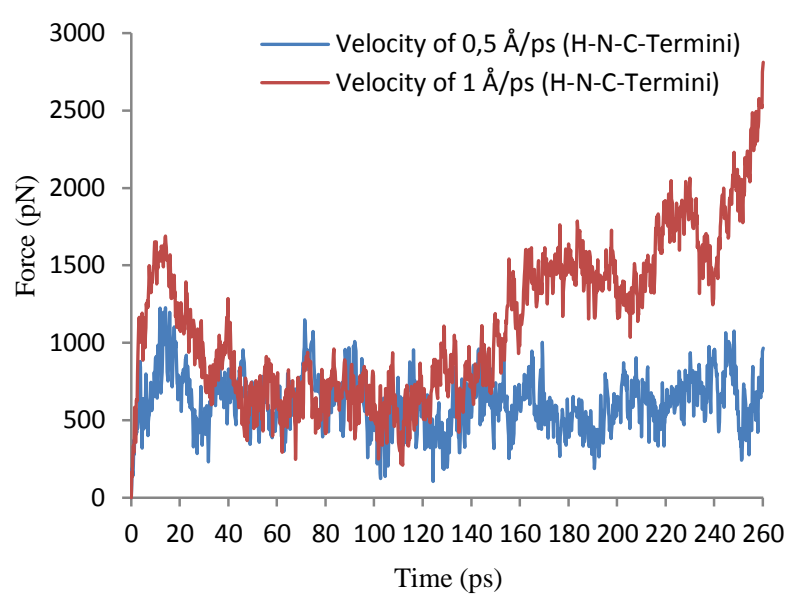

Fig. 6. Graph of force versus time in the first 260 ps SMD simulation. The blue color is a graph obtained at a constant velocity of $0.5 \AA / p s$, while the red color is a graph obtained at a constant velocity of $1 \AA$. ps.

At the initial condition, prior the SMD simulation, there were 94 hydrogen bonds present in the structure of the nimotuzumab $\mathrm{F}(\mathrm{ab})$ fragment. Hydrogen bonds occur between the residues of the heavy chain and the light chain, and inter-residues of the heavy chain and the light chain. Hydrogen bonds between residues of the heavy chain and the light chain play a significant role in the formation of a quaternary structure of the nimotuzumab $F(a b)$ fragment, which is the bond that unites the heavy chain polypeptide with a light chain polypeptide. The two chains are linked by seven hydrogen bonds as shown in Table 3. 
Table 3. Hydrogen Bonds Between the Heavy Chain (SegU) and the Light Chain (SegV)

\begin{tabular}{|c|c|c|c|c|c|}
\hline \multirow{2}{*}{ No } & \multicolumn{2}{|c|}{ Donor } & \multicolumn{2}{|c|}{ Acceptor } & \multirow{2}{*}{$\begin{array}{c}\text { Bond } \\
\text { Length } \\
(\AA)\end{array}$} \\
\hline & Residue & Atom & Residue & Atom & \\
\hline 1 & $\begin{array}{l}\text { SegU-GLN95- } \\
\text { Side }\end{array}$ & HE22 & $\begin{array}{c}\text { SegV-TYR36- } \\
\text { Side }\end{array}$ & $\mathrm{OH}$ & 1.9 \\
\hline 2 & $\begin{array}{l}\text { SegU-GLN95- } \\
\text { Side }\end{array}$ & HE21 & $\begin{array}{c}\text { SegV-ASP34- } \\
\text { Side }\end{array}$ & OD2 & 1.83 \\
\hline 3 & $\begin{array}{c}\text { SegV-GLN38- } \\
\text { Side }\end{array}$ & HE21 & $\begin{array}{l}\text { SegU-GLN39- } \\
\text { Side }\end{array}$ & OE1 & 1.76 \\
\hline 4 & $\begin{array}{l}\text { SegU-HSE164- } \\
\text { Side }\end{array}$ & HE2 & $\begin{array}{l}\text { SegV-ASP167- } \\
\text { Side }\end{array}$ & OD1 & 1.78 \\
\hline 5 & $\begin{array}{c}\text { SegV-THR164- } \\
\text { Side }\end{array}$ & HG1 & $\begin{array}{l}\text { SegU-HSE164- } \\
\text { Side }\end{array}$ & ND1 & 1.97 \\
\hline 6 & $\begin{array}{c}\text { SegV-SER162- } \\
\text { Side }\end{array}$ & HG1 & $\begin{array}{c}\text { SegU-PRO167- } \\
\text { Main }\end{array}$ & $\mathrm{O}$ & 1.77 \\
\hline 7 & $\begin{array}{c}\text { SegV-GLN160- } \\
\text { Side }\end{array}$ & HE21 & $\begin{array}{l}\text { SegU-LEU170- } \\
\text { Main }\end{array}$ & $\mathrm{O}$ & 1.91 \\
\hline
\end{tabular}

For the purpose of simulations, a hydrogen bond is defined to occur if the distance between the donor atom and acceptor atom is at most $3 \AA$ and the maximum angle formed between these two atoms is $20^{\circ}$. Figure 7 (a) shows the relationship between distances of donor and acceptor atoms and simulation time.

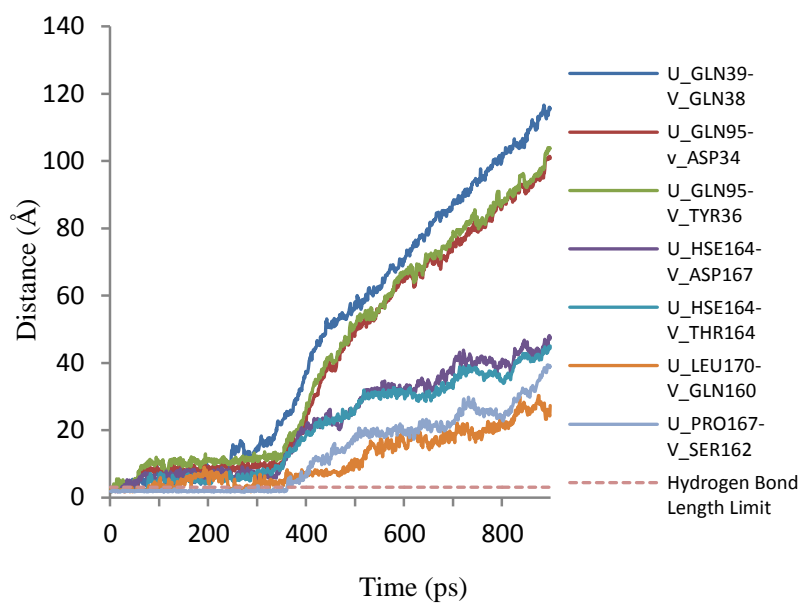

(a)

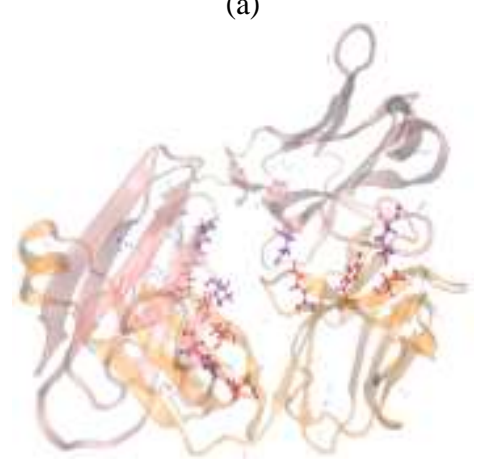

(b)

Fig. 7. (a) The distance between donor and acceptor atoms during the simulation period. A fine line is the distance between the two atoms, while the dashed line is the distance of the two atoms that still allows the hydrogen bonds, (b) Rendering of the structure before SMD simulations. Seen the 14 residues forming hydrogen bonds connecting the heavy chain and the light chain, with licorice imaging techniques, while the whole structure of nimotuzumab displayed with new cartoon imaging techniques. Figure is created using VMD 1.9 [15].
It appears that the seven hydrogen bonds linking the heavy chain and the light chain undergo rupture (distance > $3 \AA$ ) at the beginning of the simulation. The bond between residue GLN39 of the heavy chain and residue GLN38 of the light chain would break first at the simulation time of $3 \mathrm{ps,} \mathrm{followed} \mathrm{by} \mathrm{another} \mathrm{six} \mathrm{hydrogen}$ bonds (Table 4). The breaking of the hydrogen bonds will contribute to the onset of the force peak (Fig. 6) and also can trigger a process of the nimotuzumab $\mathrm{F}(\mathrm{ab})$ fragment unfolding.

Table 4. The rupture of seven hydrogen bonds between the heavy chain and the light chain. The first bond breaking occurs from top to bottom

\begin{tabular}{|c|c|c|c|c|}
\hline No & $\begin{array}{l}\text { Donor } \\
\text { Residue }\end{array}$ & $\begin{array}{l}\text { Acceptor } \\
\text { Residue }\end{array}$ & $\begin{array}{l}\text { Displacem } \\
\text { ent }(\AA)\end{array}$ & $\begin{array}{c}\text { Time } \\
\text { (ps) }\end{array}$ \\
\hline 1 & $\begin{array}{l}\text { SegV-GLN38- } \\
\text { Side }\end{array}$ & $\begin{array}{c}\text { SegU-GLN39- } \\
\text { Side }\end{array}$ & $1.76-3.10$ & 3 \\
\hline 2 & $\begin{array}{l}\text { SegU-HSE164- } \\
\text { Side }\end{array}$ & $\begin{array}{l}\text { SegV-ASP167- } \\
\text { Side }\end{array}$ & $1.78-3.76$ & 28 \\
\hline 3 & $\begin{array}{l}\text { SegV-THR164- } \\
\text { Side }\end{array}$ & $\begin{array}{l}\text { SegU-HSE164- } \\
\text { Side }\end{array}$ & $1.97-3.09$ & 34 \\
\hline 4 & $\begin{array}{l}\text { SegV-SER162- } \\
\text { Side }\end{array}$ & $\begin{array}{c}\text { SegU-PRO167- } \\
\text { Main }\end{array}$ & $1.77-3.12$ & 43 \\
\hline 5 & $\begin{array}{l}\text { SegU-GLN95- } \\
\text { Side }\end{array}$ & $\begin{array}{c}\text { SegV-TYR36- } \\
\text { Side }\end{array}$ & $1.9-4.06$ & 44 \\
\hline 6 & $\begin{array}{l}\text { SegU-GLN95- } \\
\text { Side }\end{array}$ & SegV-ASP34-Side & $1.83-3.46$ & 50 \\
\hline 7 & $\begin{array}{l}\text { SegV-GLN160- } \\
\text { Side }\end{array}$ & $\begin{array}{c}\text { SegU-LEU170- } \\
\text { Main }\end{array}$ & $1.91-3.43$ & 300 \\
\hline
\end{tabular}

Application of force to the C-terminal end of the heavy chain, which is the region close to the $\mathrm{CH} 1$ domain of the nimotuzumab $\mathrm{F}(\mathrm{ab})$ fragment, will be used for analyzing the hydrogen bonds in this domain. From three-dimensional structure analysis, the $\mathrm{CH} 1$ domain is formed by the heavy chain residues numbered 116-212. Accordingly, there are 27 hydrogen bonds in the $\mathrm{CH} 1$ domain, in which the bonds between residue LYS143 and ASP144 including between residue GLY162 and THR183 will break up first at the simulation time of 7 ps. This bond breaking is followed by the ruptures of the bond between residue TYR194 and VAL211 at 14 ps and, afterward, the bond between residue GLU148 and TYR145 at 55 ps (see Table 5). 
Table 5. The rupture of some of hydrogen bonds between residues of the heavy chain. The first bond breaking occurs from top to bottom

\begin{tabular}{|c|c|c|c|c|c|c|}
\hline \multirow{2}{*}{ No } & \multicolumn{2}{|c|}{ Donor } & \multicolumn{2}{|c|}{ Acceptor } & \multirow{2}{*}{$\begin{array}{l}\text { Distance } \\
\text { (§) }\end{array}$} & \multirow{2}{*}{$\begin{array}{c}\text { Time } \\
\text { (ps) }\end{array}$} \\
\hline & Residue & Atom & Residue & Atom & & \\
\hline 1 & $\begin{array}{l}\text { LYS143- } \\
\text { Side }\end{array}$ & HZ2 & $\begin{array}{l}\text { ASP144- } \\
\text { Side }\end{array}$ & OD1 & $\begin{array}{l}1,77- \\
3,64\end{array}$ & 7 \\
\hline 2 & $\begin{array}{c}\text { THR183- } \\
\text { Main }\end{array}$ & HN & $\begin{array}{l}\text { GLY162- } \\
\text { Main }\end{array}$ & $\mathrm{O}$ & $\begin{array}{l}1,94- \\
3,08\end{array}$ & 7 \\
\hline 3 & $\begin{array}{c}\text { VAL211- } \\
\text { Main }\end{array}$ & $\mathrm{HN}$ & $\begin{array}{c}\text { TYR194- } \\
\text { Main }\end{array}$ & $\mathrm{O}$ & $\begin{array}{l}1,79- \\
3,13\end{array}$ & 14 \\
\hline 4 & $\begin{array}{l}\text { TYR145- } \\
\text { Side }\end{array}$ & $\mathrm{HH}$ & $\begin{array}{l}\text { GLU148- } \\
\text { Side }\end{array}$ & OE1 & $\begin{array}{l}1,67- \\
3,85\end{array}$ & 55 \\
\hline
\end{tabular}

By comparing Fig. 6 and Table 5, it is apparent that there is a correlation between the force peak and the breaking of the hydrogen bonds. At the time of 14 ps simulation with a constant velocity of $0.5 \AA / \mathrm{ps}$, the first force peak is at $1226 \mathrm{pN}$. At the same time, there is a breaking of the hydrogen bond between the residues VAL211 and TYR194. It can be concluded that the force peak of $1226 \mathrm{pN}$ arises from the rupture of a hydrogen bond between these two residues. It can be presumed that the hydrogen bond between VAL211 and TYR194 residues is one of the bonds determines the stability of $\mathrm{CH} 1$ domain. After this peak is reached, the force falls back during the simulation, simultaneously along with the other hydrogen bond breakings. At the end of the simulation, the graph rises sharply until the unfolding process is complete (Fig. 8).

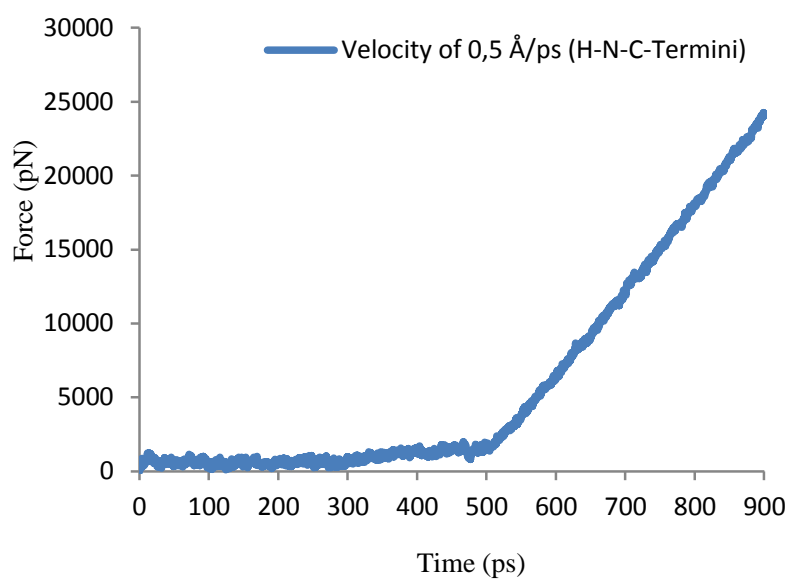

Fig. 8. Graph of force versus time for 900 ps SMD simulation.

Figure 8 shows that after $500 \mathrm{ps}$ of the SMD simulation, the force increases sharply reaching a value of $25.000 \mathrm{pN}$ at the simulation time of $900 \mathrm{ps}$. This may be due to the quaternary structure of the nimotuzumab $\mathrm{F}(\mathrm{ab})$ fragment consisting of two polypeptide chains of heavy and light chains. Due to the force applied to the $\mathrm{C} \alpha$ atoms of the C-terminal end of the heavy chain, the force works directly on the heavy chain, rather than on the light chain. Until the end of the simulation, only the heavy chain is undergoing a process of unfolding, whereas the light chain is still in the folded state, thus it results in a huge force which could cause the unfolding process in the light chain (Fig. 9). On the other hand, the results obtained from the SMD simulation with the working force at the $\mathrm{C} \alpha$ atoms of the C-terminal end of the light chain, indicate that only the light chain is undergoing a process of unfolding, whereas the heavy chain is still in the folded state (Fig. 10). Supposing an analogy to the contrary process, the possibility of the folding process of the nimotuzumab $\mathrm{F}(\mathrm{ab})$ fragment is initiated with folding on each heavy and light chain, in which then both chains are joined together to form a quaternary structure of the nimotuzumab $\mathrm{F}(\mathrm{ab})$ fragment.

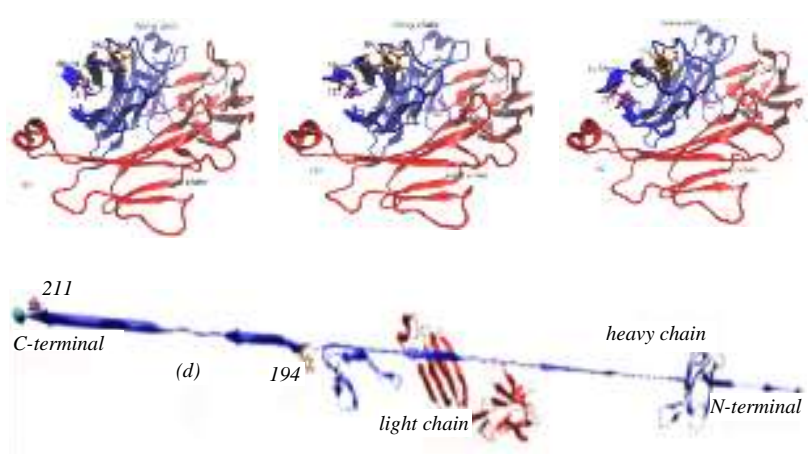

Fig. 9. The process of unfolding and breaking of hydrogen bonds during the SMD simulation with a pulling velocity of 0.5 $\AA / p s$. The blue color is a heavy chain and the red color is a light chain. (a) For 0 ps, there is a hydrogen bond between the TYR194 and VAL21 residues as shown by the green dashed lines, (b) For $7 \mathrm{ps}$, the hydrogen bond starts to rupture, (c) At $14 \mathrm{ps}$ simulation, there is no presence of hydrogen bond, (d) At 900 ps simulation, the heavy chain has undergone a process of unfolding, whereas the light chain is still in a folded state.

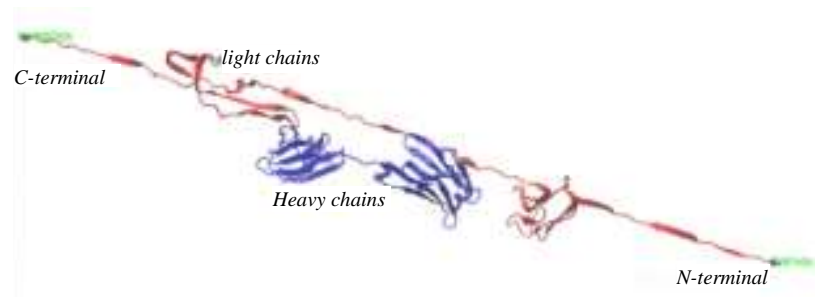

Fig. 10. The results of the SMD simulation for the light chain. The blue color is the heavy chain and the red color is the light chain. At 900 ps simulation, the light chain has undergone a process of unfolding, whereas the heavy chain is still in a folded state. 
The force peak of $1226 \mathrm{pN}$ in the process of unfolding the nimotuzumab $\mathrm{F}(\mathrm{ab})$ fragment delineates a protein with a molecular weight of $50 \mathrm{kDa}$ that is relatively small when compared with the actual molecular weight. According to Hui $\mathrm{Lu}$ and Klaus Schulten [16], the force needed to pull and lead the unfolding of a protein depends on the architecture of the proteins.

The protein architecture takes account of three-dimensional conformation of secondary structure, the position of $\beta$-strand structure (parallel or not parallel), and the presence or absence of a hydrogen bonds between the N-terminal and C-terminal, either directly or indirectly. These will affect the magnitude of force that is required to trigger the protein unfolding process.

There are three classes of proteins according to Hui $\mathrm{Lu}$ and Klaus Schulten [16], i.e. class Ia, class Ib and class II. In the structure of class Ia, hydrogen bonds connect directly between the N-terminal and C-terminal, which is laid out in the same direction. It would provide the best protection against the unfolding process because it requires breaking a larger number of hydrogen bonds simultaneously to unfold the protein molecule, so it will necessary need a large force. In the structure of class $\mathrm{Ib}$, hydrogen bonds do not connect directly between the $\mathrm{N}$-terminal and $\mathrm{C}$-terminal. The hydrogen bond breakings will occur in a wider place, so it will be a gradual increase in force. Whereas in structure of class II, hydrogen bonds connect directly between the N-terminal and C-terminal, which is laid out in opposite directions. The force required to trigger the process of unfolding is smaller and not enough to cause extreme peak. Hydrogen bonds will rupture one by one so it does not need a great force. Because of the SMD simulations of the nimotuzumab $F(a b)$ fragment were done with work force at the C-terminal end of the heavy chain as part of a region of the $\mathrm{CH} 1$ domain, whereas the $\mathrm{N}$-terminal end was fixed, the $\mathrm{CH} 1$ domain has an architecture-like structure of class $\mathrm{Ib}$ as indicated from the results of structural analysis using VMD 1.9 program [15]. In this structure, there are no hydrogen bonds connecting directly between the $\mathrm{N}$-terminal strand and C-terminal strand. However, the hydrogen bonds occurs between the terminal strand and nonterminal strand. The position of the $\mathrm{N}$-terminal and C-terminal of $\mathrm{CH} 1$ domain is also located in the opposite direction (see Fig. 11).

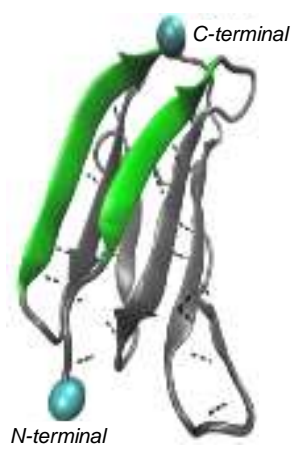

Fig. 11. Architecture of the $\mathrm{CH} 1$ domain resembles classes $\mathrm{Ib}$ made by Hui $\mathrm{Lu}$ and Klaus Schulten [16]. The green color depicts the $\mathrm{N}$-terminal and C-terminal strands. This figure is created using VMD 1.9 [15].

In addition to the SMD simulations performed at a constant velocity of $0.5 \AA / p s$, simulations were also carried out with the pulling velocity of $1 \AA / \mathrm{ps}$ and resulted in force peak of $1688 \mathrm{pN}$ at 14 ps. This force is greater than that of a velocity of $0.5 \AA / \mathrm{ps}$. Therefore, the force required to cause the unfolding process of the nimotuzumab $F(a b)$ fragment using atomic force microscopy (AFM) is predicted to be smaller, because of the pulling velocity used by SMD simulations is larger than the AFM. In SMD simulation with a pulling velocity of $0.5 \AA / \mathrm{ps}$, a force peak of $1226 \mathrm{pN}$ was obtained. In addition, from SMD simulations at a constant velocity of $0.5 \AA / \mathrm{ps}$, the rupture of the hydrogen bond between the residue TYR194 and residue VAL211 occur after 14 ps simulation or after a 7-Å lengthening.

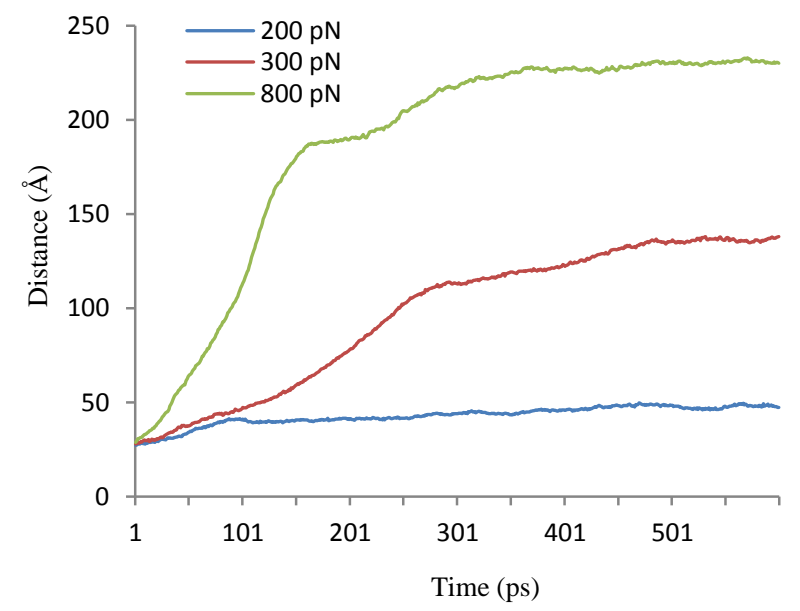

Fig. 12. Graph of N-terminal and C-terminal distance versus time

Further, from the SMD simulation using second protocol with contstant force, it as obtained that the rupture of the hydrogen bond between these two residues, TYR194 and VAL211, occurred at $484 \mathrm{ps}, 29 \mathrm{ps}$ and $1 \mathrm{ps}$ for the working forces of $200 \mathrm{pN}, 300 \mathrm{pN}$ and $800 \mathrm{pN}$, respectively (Fig. 12). 
It can be concluded that in the actual experiment (AFM), the force required to trigger the unfolding process of $\mathrm{CH} 1$ domain of the heavy chain of the nimotuzumab $\mathrm{F}(\mathrm{ab})$ fragment ranges from $300 \mathrm{pN}$ to $800 \mathrm{pN}$.

\section{CONCLUSION}

We have performed molecular dynamics simulations of the nimotuzumab $\mathrm{F}(\mathrm{ab})$ fragment for various temperatures and steered molecular dynamics (SMD) simulation at a constant velocity of $0.5 \AA / p s$. It can be concluded that temperature changes have a considerable effects on the conformation of both heavy and light chains of the nimotuzumab $F(a b)$ fragment, especially at residues numbered 27 (tyrosine), 28 (threonine), 83 (arginine), 84 (serine), 98 (tryptophan), 99 (phenylalanine), 183 (threonine), 184 (valine), and 185 (proline) for the heavy chain and 1 (aspartate), 127 (serine), and 186 (tyrosine) for the light chain, respectively. The residues numbered 98 and 99 are part of CDR3, however, the conformational changes of residues in the CDR1 and CDR2 regions are much lower. Based on the SMD simulations, the hydrogen bond between residue VAL211 and TYR194 determines the stability of $\mathrm{CH} 1$ domain of the nimotuzumab $\mathrm{F}(\mathrm{ab})$ fragment.

For further research, it is advisable to perform molecular dynamics simulations of immunoconjugates of radiolabeled nimotuzumab and their interactions with EGFR in blood plasma at body temperature.

\section{ACKNOWLEDGMENT}

The author would like to thank Hasan $\mathrm{Al}$ - Rashid for any advice and assistance during the research, and the Centre for Education and Training of the National Nuclear Energy Agency of Indonesia (BATAN) which has provided financial assistance for this study. The authors also like to thank all those who have given advice, motivation and support both materially and spiritually, so that this research can be completed.

\section{REFERENCES}

1. M. Takeda, I. Okamoto, Y. Nishimura, et al., Lung Cancer: Target and Therapy 2 (2011) 59.

2. M.O. Rodriguez, T.C. Rivero, B.R. del Castillo, et al., Cancer Biology and Therapy 9 (2009) 343.

3. A.M. Scott, J.P. Allison and J.D. Wolchok, Cancer Immunity 12 (2012) 14.

4. Y. Akashi, I. Okamoto, T. Iwasa, et al., British Journal of Cancer 98 (2008) 749.

5. T. Crombet, M. Osorio, T. Cruz, et al., Journal of Clinical Oncology 22 (2004) 1645.

6. M.S. Ramakrishnan, A. Eswaraiah, T. Crombet, et al., mAbs 1 (2009) 41.

7. S. Li, K.R. Schmitz, P.D. Jeffrey, et al., Cancer Cell. 7 (2005) 301.

8. J.A. Katzel, M.P. Fanucchi and Z. Li, Journal of Hematology and Oncology 2 (2009) 1.

9. G. Speake, B. Holloway and G. Costello, Curr. Opin. Pharmacol. 5 (2005) 343.

10. A. Talavera, R. Friemann, S.G. Puerta, et al., Cancer Res. 69 (2009) 5851.

11. D.G.P. Allan, The Oncologist 10 (2005) 760.

12. R. Perez, E. Moreno, G. Garrito, et al., Cancers 3 (2011) 2014.

13. T.S. Humani, M. Ramli, C.T. Rustendi, et al., Preparation and Stability Test of 177Lu-DOTA-Nimotuzumab as a Radioimmunotherapy of Cancer, Proceedings of National Seminar on Human Resources of Nuclear Technology VI (2010) 663. (in Indonesia)

14. J.C. Phillips, R. Braun, W. Wang, et al., J. Comput. Chem. 26 (2005) 1781.

15. W. Humphrey, A. Dalke and K. Schulten, J. Mol. Graphics 14 (1996) 33.

16. H. Lu and K. Schulten, Proteins: Struct., Funct., Genet. 35 (1999) 453. 